\title{
Combining curriculum vitae and bibliometric analysis: mobility, gender and research performance
}

\author{
Ulf Sandström
}

\begin{abstract}
This paper demonstrates the benefits of combining curriculum vitae studies with advanced bibliometrics. Based on data from $326 \mathrm{CVs}$ within one broad medical subject area we perform a cluster analysis of CV data. Data reduction produces four different groups of scientists: 1) mobile, 2) immobile, 3) excellent and 4) entrepreneurial. While it is clear that the most mobile and the least mobile researchers represent opposites also in citation performance we should acknowledge that for the large majority, with a low and medium mobility, there is no linear pattern of performance. The paper points at a double process where there are on the one hand selection processes at universities picking out 'the winners' and on the other hand self selection processes where researchers enhance their own performance by being mobile.
\end{abstract}

I N RECENT YEARS, curricula vitae (CVs) have been identified as an interesting source for research with different approaches to the study of science and technology. Pioneering work was done by the Research Value Mapping Program (Dietz et $a l, 2000)$ showing that CVs have great potential as data for research on research but that there are a number of methodological problems that have to be tackled. These include issues such as availability of CVs, and accuracy and coding of CVs. Several CV studies have been presented (Dietz and Bozeman, 2005; Gaughan and Robin, 2004; Gaughan and Bozeman, 2002; Lee and Bozeman, 2005), and other groups have joined this research agenda (e.g. Mangematin, 2000, 2001; Cañibano, 2008).

This paper discusses the combination of $\mathrm{CV}$ data with bibliometric data. A first attempt to use both was reported by Lee and Bozeman (2005) in their

Docent and associate professor Ulf Sandström is with the Dept of Social Change and Culture at Linköping University, 58183 Linköping, Sweden; Email: ulfsa@isak.liu.se; Tel: +46708137376.

Support from FORMAS for the research project 151-2007159 is acknowledged. The author would like to express his gratitude to Ulf Heyman, Martin Hällsten, Erik Sandström, and one anonymous referee for their valuable remarks. important paper on research collaboration. There is a strong relationship between collaboration and productivity, but it is hard to figure out the exact relationship due to a number of methodological considerations:

1. There are several ways of accounting for collaboration, for example, normal counts (all publications counted as whole counts) and fractional counts (dividing credit by the number of coauthors).

2. Researcher status (e.g. professor or not professor) is of importance.

3. Field differences have to be taken into account.

When Lee and Bozeman (2005) used fractional counts, collaboration was found to be unrelated to productivity, but with normal counts collaboration was a strong predictor. Even though the 'simple problem' of collaboration and productivity has been discussed intensively, there are still several aspects that could be added. One aim of this paper is to bring citations into the analysis and to look for other roads to understand the multifaceted problems of collaboration, rank, age and productivity. Another aim is to test clustering analysis as a data reduction method. A third aim is to focus on mobility and 
combine that factor with citation analysis and clustering techniques. Lastly, it is interesting to include gender aspects (Cole and Zuckerman, 1984; Prpic, 2002) in the analysis.

\section{Data and methodological issues}

The primary data used in this analysis come from the CVs of a population consisting of all senior scientists who were positioned as grant holders at a Swedish research foundation by June 2005, in all 326 researchers. The research area can be described in terms of medical, both preclinical and clinical, research. The population consists of principal investigators (PIs). These PIs received grants during 20022004; a normal grant lasts for a three-year period. The average grant was approximately $€ 60,000$ per year.

The CVs were collected in the period SeptemberOctober 2005 with the specific aim of performing a bibliometric study of the population. The manual coding used a template available on the Internet $<$ www.forskningspolitik.se/cv>, which was developed especially for this dataset.

While field differences are an issue in studies using data from multiple areas and disciplines of science, there is more common ground for comparison in this study. However, even in this case - medical research - it is necessary to consider differences between clinical and non-clinical research (Narin and Hamilton, 1996). Probably there are differences regarding output variables such as number of publications and number of citations. Therefore, we use a field-normalization method (Glänzel, 1996; Van Raan, 2004) in our citation analysis and the main contribution of this paper is to demonstrate that $\mathrm{CV}$ analysis using this method is stronger methodologically than straight paper or citation counts.

\section{Methodological issue 1: quality of CVs}

The problem in this investigation, as in many other CV studies (Dietz et al, 2000), is the shifting quality of information. Many CVs are condensed or 'truncated'. The collection of CVs was done to enable a bibliometric study, which indicated that appointments and publications were needed. Accordingly, not all the personal information was given by all individuals.

But even a short résumé or $\mathrm{CV}$ can be used to get hold of important data (see Table 1) if it is used together with other sources of information (Internet, CVs from other agencies, etc.). The dataset consists of approximately 80 variables of which 26 are considered as interesting in this context. Less than one third of all CVs give information about family status and number of children. Parental leave (number of months) is the type of information where we unfortunately are unaware of the completeness of data.
Table 1. List of variables with descriptive statistics

\begin{tabular}{|c|c|c|c|c|c|}
\hline Variable & $\mathbf{N}$ & Min & $\operatorname{Max}$ & Mean & $\begin{array}{l}\text { Std. } \\
\text { Dev. }\end{array}$ \\
\hline Age (in 2005) & 326 & 33 & 76 & 49.48 & 8.697 \\
\hline Marital status & 326 & 0 & 3 & 0.92 & 1.360 \\
\hline No. of children & 106 & 1 & 5 & 2.43 & 0.805 \\
\hline Parental leave (month) & 20 & 0 & 36 & 17.7 & 11.921 \\
\hline Gender & 326 & 0 & 1 & 0.77 & 0.420 \\
\hline National origin & 326 & 1 & 3 & 1.15 & 0.533 \\
\hline Chapters and reviews & 116 & 1 & 64 & 8.17 & 8.554 \\
\hline Articles total & 326 & 0 & 760 & 106.50 & 115.70 \\
\hline Patents total & 326 & 0 & 25 & 0.55 & 2.118 \\
\hline PhD supervised total & 209 & 1 & 40 & 7.49 & 6.923 \\
\hline PhD 1998-2005 & 180 & 1 & 40 & 4.48 & 4.070 \\
\hline Postdoctors supervised & 324 & 0 & 50 & 1.86 & 4.764 \\
\hline Postdoctors 1998-2005 & 88 & 1 & 50 & 5.60 & 6.373 \\
\hline Basic grants & 103 & 1 & 10 & 2.83 & 1.953 \\
\hline Strategic grants & 35 & 1 & 5 & 2.06 & 0.873 \\
\hline Applied grants number & 46 & 1 & 7 & 2.07 & 1.162 \\
\hline $\begin{array}{l}\text { Entrepreneurial } \\
\text { achievement }\end{array}$ & 326 & 0 & 13 & 0.50 & 1.452 \\
\hline Editorial appointments & 326 & 0 & 16 & 0.77 & 2.059 \\
\hline issions of trust & 110 & 1 & 10 & 4.13 & 2.362 \\
\hline Committees & 104 & 1 & 20 & 4.84 & 3.184 \\
\hline Honors and awards & 94 & 1 & 28 & 2.48 & 3.155 \\
\hline Visiting professorships & 18 & 1 & 5 & 1.94 & 1.349 \\
\hline Year of PhD & 325 & 1958 & 2003 & 1987 & 8.6 \\
\hline $\begin{array}{l}\text { Year of docent } \\
\text { (associate) }\end{array}$ & 262 & 1961 & 2005 & 1992 & 9.9 \\
\hline Year of professor & 167 & 1968 & 2005 & 1996 & 7.352 \\
\hline Mobility & 321 & 0 & 3 & 0.470 & 0.632 \\
\hline
\end{tabular}

Note: $\mathrm{N}=326$, most variables are counted as number of

Swedish CVs do not commonly report all grants received, although it seems to become important for the younger population. About a third of the CVs hold relevant data on research income. Of course, there is information on university, disciplinary background, research area, and all appointments, with the year of appointment. The latter data will be used in a section below that will demonstrate how this information can be used.

\section{Methodological issue 2: coding practice}

The material was coded by the author. The first 25 CVs were examined and a learning process was started. After a while most of the problems were known and a strategy was outlined to handle these problems.

Instead of coding each patent, entrepreneurial activity, editorial appointment, committee, etc. it was decided to use a more time-efficient model. As we have a parallel process of doing bibliometrics we found that it was not necessary to collect all information on each publication.

\section{Methodological issue 3: data reduction}

Cluster analysis is a generic term for a data reduction based on multivariate data analysis (Tjissen and de Leuuw, 1988). The grouping of objects of similar kind into categories is done with a hierarchical 
clustering technique in SPSS (Ward's method). Thirteen of the variables consist of more or less complete data (age, total articles, patents, PhDs supervised, postdocs supervised, entrepreneurial achievements, editorial appointments, year of $\mathrm{PhD}$, and mobility) and the following analysis is based on these variables. Four different clusters were identified (see Table 2).

Looking deeper into the result of the cluster analysis we find that there are four different groups. To a large extent the first two - Group 1 and Group 2 - are identical. They are the same age, have the same number of articles, and so on. But, in one aspect they differ significantly: mobility. In the process of building a career the first group seems to have been looking around for new positions and new possibilities. It is notable that this type of mobility does not pay off in normal types of recognition (awards, etc.). The first group might be called the mobile scientists.

The second group might be used as reference values for contrasting the behaviour of other groups, i.e. they are what we would call immobile scientists. This is the largest cluster of researchers and fairly close to the mean of all groups. The standard deviation is high for many variables, but as 'immobile scientists' they seem to consist of less productive, less entrepreneurial workers, and have a slower career track from $\mathrm{PhD}$ to professorship.

The last two, Group 3 and Group 4, are smaller in size, but they have interesting common features. In many ways the third group has the highest forms of excellence as measured by number of papers. Accordingly, they are distinguished by their low entrepreneurial activity as there is a 'crowding out'-effect of patent-seeking activities; entrepreneurs cannot write as many papers as researchers who concentrate on science. The third group is the oldest compared to the others and they can be characterized as highly productive, producing approximately 70 papers over the period of seven years - 10 papers per year. This high productivity is explained by their supervising activities as they tend to have the highest figures for postdocs and $\mathrm{PhDs}$ under supervision. The group is fairly large - 37 cases - and they are very highly appreciated by colleagues and by society. Having high figures on both awards and editorial appointments, they can be said to conduct the role of academic 'watchdogs' controlling vital resources like positions (PhDs and postdocs) and journals. The third group is called the excellence group.

The fourth group is a selection of excellent researchers with high productivity, a dedicated patenting and entrepreneurial activity, many editorial appointments and a fast career from $\mathrm{PhD}$ to full professor. The latter is seen from the career variable, which is the number of years from $\mathrm{PhD}$ to the full professorship. They are older than average and they have had several opportunities to move around: this is obvious from mobility data, which show the number of times they have changed their affiliation during their career (not counting the postdoc period). The fourth group is called the entrepreneurial group.

\section{Methodological issue 4: research impact}

As indicated above, publication data on each of the 326 researchers were collected from the Thomson/ ISI Internet Web of Science database. Data on all papers were downloaded using manual techniques for bibliometric identification. Additionally, to build up reference values, data on all journal articles in all subject categories where these researchers have been publishing were downloaded. Using the ISI UT number as the identifier we connected the author

Table 2. Cluster analysis (four groups) and variable mean values per group

\section{Cluster}

\begin{tabular}{|c|c|c|c|c|c|c|c|c|c|c|c|c|c|}
\hline 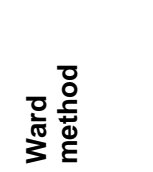 & 於 & 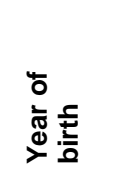 & 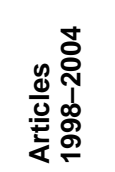 & 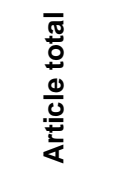 & 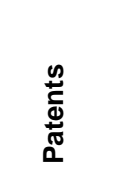 & 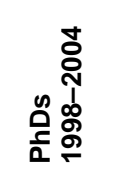 & 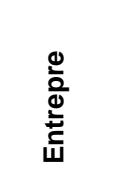 & 픔 & $\frac{n}{\frac{0}{0}}$ & 仓ัँ & 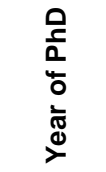 & 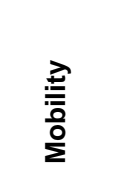 & 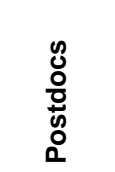 \\
\hline 1. Mean & 0.759 & 56.111 & 27.769 & 87.352 & 0.648 & 4.079 & 0.083 & 0.306 & 2.033 & 13.585 & 1988.4 & 1.093 & 1.009 \\
\hline $\mathrm{N}$ & 108 & 108 & 108 & 108 & 108 & 63 & 108 & 108 & 30 & 53 & 108 & 108 & 108 \\
\hline Std. Dev & 0.43 & 8.49 & 21.28 & 77.04 & 1.71 & 2.93 & 0.28 & 0.97 & 1.13 & 4.33 & 7.98 & 0.44 & 2.11 \\
\hline 2. Mean & 0.755 & 56.246 & 24.350 & 88.678 & 0.126 & 3.408 & 0.119 & 0.364 & 2.250 & 15.179 & 1988.8 & 0.000 & 0.455 \\
\hline $\mathrm{N}$ & 143 & 142 & 143 & 143 & 143 & 71 & 143 & 143 & 32 & 56 & 143 & 143 & 143 \\
\hline Std. Dev & 0.43 & 8.69 & 16.36 & 85.33 & 0.53 & 2.05 & 0.38 & 0.96 & 1.87 & 4.20 & 8.70 & 0.00 & 1.29 \\
\hline 3. Mean & 0.811 & 50.297 & 70.973 & 247.838 & 0.081 & 7.630 & 0.405 & 3.027 & 5.231 & 13.265 & 1981.2 & 0.378 & 9.838 \\
\hline $\mathrm{N}$ & 37 & 37 & 37 & 37 & 37 & 27 & 37 & 37 & 13 & 34 & 37 & 37 & 37 \\
\hline Std. Dev & 0.40 & 6.92 & 62.75 & 191.33 & 0.36 & 7.52 & 0.80 & 3.86 & 7.36 & 3.79 & 6.33 & 0.68 & 9.63 \\
\hline 4. Mean & 0.931 & 56.207 & 43.069 & 164.345 & 3.034 & 6.071 & 4.276 & 1.828 & 1.684 & 11.857 & 1984.7 & 0.621 & 2.207 \\
\hline $\mathrm{N}$ & 29 & 29 & 29 & 29 & 29 & 14 & 29 & 29 & 19 & 21 & 29 & 29 & 29 \\
\hline Std. Dev & 0.26 & 9.88 & 31.10 & 147.47 & 5.59 & 4.34 & 2.55 & 3.46 & 0.82 & 4.08 & 10.11 & 0.73 & 4.39 \\
\hline Total Mean & 0.779 & 55.500 & 32.669 & 113.726 & 0.565 & 4.514 & 0.521 & 0.789 & 2.479 & 13.841 & 1987.4 & 0.473 & 1.899 \\
\hline $\mathrm{N}$ & 317 & 316 & 317 & 317 & 317 & 175 & 317 & 317 & 94 & 164 & 317 & 317 & 317 \\
\hline Std. Dev & 0.42 & 8.72 & 32.08 & 119.34 & 2.14 & 4.11 & 1.47 & 2.08 & 3.15 & 4.26 & 8.70 & 0.63 & 4.81 \\
\hline
\end{tabular}


name to author names in the downloaded ISI data. This gave analytical results from a SQL database in the form of exact author parts of articles (author fractionalized articles).

We apply the bibliometric methods put forward by, for example, the Leiden and Leuven groups (Glänzel, 1996; Van Raan, 2004). The methods for normalization take all the relevant information that follows with the bibliometric data into account. Each journal in the Thomson/ISI database is assigned to one or several sub-fields (e.g. microbiology, biochemistry and molecular biology). Our analysis focuses on citations per paper (CPP) in relation to journal citation score (JCS) and CPP in relation to field citation score (FCS). In both cases the mean citation score for the journal set is the point of reference for the calculations (Van Raan, 2004, 2006). Self-citations are included and we apply an open citation window. Articles from 1998 are compared to all articles from 1998, etc. Nederhof and Visser (2004) have shown why an open window gives a methodological advantage.

In all, the 326 PIs produced 7,907 unique articles. The bibliometric analysis is based on citations up until May 2007 to articles published during the period 1998-2004.

\section{Results}

The CV material is interesting because the distribution over age-classes is fairly even. There are 50-60 in each of six age-classes $(<41 ; 41-45$; etc.), so the representation of young researchers as well as midaged and seniors is satisfactory.

At the overall level, relative citations per field are $47 \%$ better than world average (see Table 3 ). The citation analysis reveals that differences between the groups are quite small; a variation around $10 \%$ is probably not significant. In the journals where all these researchers publish, they are $15 \%$ more successful in gaining recognition (citations from their colleagues) than the global averages.

A closer look shows that the differences might relate to the quality of journals. While the mobility

Table 3. Journal and field-normalized bibliometric values per cluster group, and standard deviation

\begin{tabular}{lrrrrr}
\hline Ward & $\begin{array}{c}\text { No. } \\
\text { cases }\end{array}$ & $\begin{array}{c}\text { CPPI } \\
\text { JCS }\end{array}$ & $\begin{array}{c}\text { JCSI } \\
\text { FCS }\end{array}$ & $\begin{array}{c}\text { CPPI } \\
\text { FCS }\end{array}$ & $\begin{array}{c}\text { Std } \\
\text { dev. }\end{array}$ \\
1. Mobility & 105 & 1.17 & 1.33 & 1.49 & 0.859 \\
2. Immobility & 141 & 1.11 & 1.28 & 1.40 & 0.758 \\
3. Excellence & 37 & 1.22 & 1.38 & 1.55 & 0.823 \\
4. Entrepreneur & 29 & 1.19 & 1.38 & 1.64 & 0.643 \\
Grand total & $\mathbf{3 1 7}$ & $\mathbf{1 . 1 5}$ & $\mathbf{1 . 3 2}$ & $\mathbf{1 . 4 7}$ & $\mathbf{0 . 7 8 2}$ \\
\hline
\end{tabular}

Notes: $\quad \mathrm{CPP} / \mathrm{JCS}=$ journal-normalized citation score CPP/FCS $=$ field-normalized citation score

\section{While male researchers tend to produce as many papers as possible, a few of which will become much cited and not so few that will be zero cited, female scientists are more prudent with their publications}

group and the immobility group tend to publish in lower-ranked journals, the excellence and entrepreneurial groups tend to publish in higher-ranked journals or journals that yield a better result in citations.

These differences are mirrored and extended in the field indicator CPP/FCS, which reveal a higher citation rate or field-related performance. As shown in Table 3, the standard deviation is about the same for the different groups. This can be interpreted as a stable variation within the groups, which points in the direction of a systematic variation between groups.

There are 73 female and 252 male researchers in the data set. Female researchers are, on average, about three years younger than their male colleagues. What are their respective performances when measured with field-normalized methods? As in several other studies, female researchers taken as a group are doing better (Long, 1992; Xie and Shauman, 1998, Sandström and Hällsten, 2009). Female researchers receive a field-normalized citation score of 1.53 and male researchers receive a slightly lower figure of 1.45 . Citation data are skewed and the analysis shows that the distributions are not exactly the same between the two gender groups. The trimmed mean (5\%) for females is 1.31 and for males 1.22.

The range of citations performance is much wider in the male group and we can explain this with differences in publication behaviour. While male researchers tend to produce as many papers as possible, a few of which will become much cited and not so few that will be zero cited, female scientists are more prudent with their publications and the citation picture seems to mirror this with fewer highly cited as well as fewer not cited articles.

From Table 4 we draw the conclusion that female principal investigators are clustered to a lesser extent in the excellence and entrepreneur groups.

The results indicate that female principal investigations in the mobile and immobile groups are getting higher citation scores. This might be explained by their lack of academic experience due to a lower age but, of course, it probably also reflects hierarchical structures within the academic community at large. 
Table 4. Field-normalized citation score (CPP/FCS) per gender and cluster group

\begin{tabular}{lrrrrrr}
\hline Group & Female & N & Male & N & Total & $\begin{array}{c}\text { Grand } \\
\text { total }\end{array}$ \\
Mobility & 1.58 & 26 & 1.46 & 79 & 1.49 & 105 \\
Immobility & 1.59 & 34 & 1.34 & 107 & 1.40 & 141 \\
Excellence & 1.09 & 7 & 1.65 & 30 & 1.55 & 37 \\
Entrepreneur & 1.16 & 2 & 1.68 & 27 & 1.64 & 29 \\
Total & $\mathbf{1 . 5 3}$ & $\mathbf{6 9}$ & $\mathbf{1 . 4 5}$ & $\mathbf{2 4 3}$ & $\mathbf{1 . 4 7}$ & $\mathbf{3 1 2}$ \\
\hline
\end{tabular}

\section{Collaboration and productivity}

A thorough investigation of the relations between collaboration and productivity would need full data on actual funding (grants). Probably, the distribution of research funding is uneven and a few in the upper end of the spectrum hold a good share of the money while the majority have to live with ordinary funding arrangements (Cesaroni and Gambardella, 2003). There seem to be a Matthew Effect (Merton, 1968) in research policy: if you have funding from one source your chances of getting funded from other sources will be higher. Research group size differs considerably according to the level of funding and if you have large resources you might even invest in facilities that open up possibilities for more collaboration with other researchers wanting to use your equipment. A plausible hypothesis would be that some of the 'best' researchers are capable of assembling large resources and that this is a forgotten explanation as to why some are more 'productive' than others. This is dealing with spurious relations as we cannot know whether the productivity was there before the funding.

Our dataset does not include actual research funding or the composition of resources. Data on this aspect are often too weak to be used for a detailed analysis (cf. Lee and Bozeman, 2005). Instead, we propose that our cluster analysis could act as a proxy for this aspect. Looking more closely at the analysis there are several indications that the excellence group, which seem to be more fortunate in several respects, could be seen as a more resourceful group. They have more PhD students, they have more postdocs, they receive a higher number of awards, and consequently they have a higher number of publications. This is confirmed by an analysis of cluster groups in relation to publications, both normal and fractional counts. Table 5 shows that there are big differences: first, between status groups and second, between cluster groups in their productivity. Among these different groups the Excellence group has the highest number of fractional counts and normal counts as well. They are almost twice as productive as the Groups 1 and 2.

In Table 6 we display differences in number of years since receiving the $\mathrm{PhD}$. While professors
Table 5. Normal counts and fractional counts per cluster and status group, mean values per author, 1998-2004

\begin{tabular}{lrrrrrr}
\hline & \multicolumn{3}{l}{ Professors } & \multicolumn{4}{c}{ Others } \\
\cline { 2 - 8 } Group & $\begin{array}{l}\text { Normal Frac. } \\
\text { count }\end{array}$ & count & & $\begin{array}{c}\text { Normal Frac. } \\
\text { count } \\
\text { count }\end{array}$ & N \\
Mobility & 37.40 & 8.6 & 52 & 18.49 & 3.9 & 53 \\
Immobility & 30.82 & 6.4 & 57 & 18.70 & 4.1 & 85 \\
Excellence & 69.68 & 15.3 & 34 & 71.67 & 17.4 & 3 \\
Entrepreneurs & 51.57 & 11.1 & 21 & 19.75 & 4.4 & 8 \\
Totalt & $\mathbf{4 3 . 6 2}$ & $\mathbf{9 . 5}$ & $\mathbf{1 6 4}$ & $\mathbf{1 9 . 7 4}$ & $\mathbf{4 . 3}$ & $\mathbf{1 4 9}$
\end{tabular}

Note: Pearson correlation between normal and frac. count is 0.93

have a higher number of papers per period (i.e. higher productivity), the non-professors have almost the same productivity no matter the length of time since they received their $\mathrm{PhD}$. This illustrates the hypothesis that you need professorial status to assemble resources for productivity. Another aspect is that productivity does not always imply a higher field-normalized citation score. The so-called 'crown indicator' is more or less unrelated to productivity. More highly cited papers seem to be written by younger researchers regardless of whether they are professors or not. This is stable even when controlled for by gender. The burden of high productivity seems to be that relative citations become a bit lower, which is reasonable as professors have to collaborate with all their staff. In conclusion, empirical data supports the view that productivity is related to professorial status, but at the same time there is evidence indicating that the most innovative research originates from younger researchers who received their PhDs in the 1990s.

Gender makes a difference in productivity (data not shown here). Female researchers have a significantly lower number of papers in their respective groups, but it should be noted that among professors

Table 6. Fractional counts per group of years since PhD (mean values per author, 1998-2004) and fieldnormalized citations score

\begin{tabular}{lcccccc}
\hline & \multicolumn{2}{c}{ Professors } & \multicolumn{2}{c}{ Others } & \multicolumn{2}{c}{ N } \\
\cline { 2 - 7 } Years since & $\begin{array}{c}\text { Frac. } \\
\text { PhD }\end{array}$ & $\begin{array}{c}\text { CPPI } \\
\text { P }\end{array}$ & $\begin{array}{c}\text { Frac. } \\
\text { P }\end{array}$ & $\begin{array}{c}\text { CPPI } \\
\text { PCS }\end{array}$ & Prof. & Others \\
B (>29) & 12.5 & 1.17 & 3.8 & 0.82 & 29 & 1 \\
C (25-29) & 11.7 & 1.39 & - & - & 31 & - \\
D (20-24) & 8.0 & 1.43 & 4.2 & 1.03 & 42 & 6 \\
F (15-19) & 8.9 & 1.37 & 5.5 & 1.20 & 36 & 20 \\
G (10-14) & 7.1 & 1.68 & 4.7 & 1.28 & 27 & 53 \\
H (5-9) & 7.5 & $>3.00$ & 3.8 & 1.68 & 2 & 65 \\
I (>5) & - & - & 2.2 & 1.82 & - & 8 \\
Total & $\mathbf{9 . 5}$ & $\mathbf{1 . 4 6}$ & $\mathbf{4 . 3}$ & $\mathbf{1 . 4 6}$ & $\mathbf{1 6 7}$ & $\mathbf{1 5 3}$ \\
\hline
\end{tabular}


there are slightly smaller variations between male and female researchers.

\section{Researcher mobility}

The advantage of using CVs is that they facilitate the study of mobility issues. They give information on all shifts from one place to another and from one position to the other. Data on these aspects seem to be more complete than other aspects, although we cannot be sure that every move during a career is reported. We have in total 1,472 moves, but not all of these represent mobility from one institution to another, as we also record all changes in position (appointments). The mobility between institutions can be counted to a total of 594, which indicates that, on average, researchers have almost two interinstitutional moves during their career. Of course, as discussed above the distribution of mobility is uneven and some researchers are mobile while others are less mobile.

If we group all researchers by low, medium and high mobility, we find that the most mobile researchers, a small group (18 persons), have the highest field citations score $(=1.80)$, but the difference between low and medium is not that significant (low $=1.42$; medium $=1.50$ ). But we want to know more about the flows of people, how are they mobile? Table 7 shows the mobility patterns from one institution (row) to another institution (column). Although postdoc experience is of interest in this context, we have decided not to include that information. Postdoc mobility can be seen as a form of 'institutionalized' mobility, valuable as such but with a specific logic. Each instance of mobility is registered as a change from row to column.

There are 15 moves from UU (Uppsala University) to KI (Karolinska Institute). There are 50 moves from UU and only 31 researchers coming to Uppsala. We could interpret this figure in different ways, but it seems obvious that Uppsala is a supplier of competence, while $\mathrm{KI}$ is a demanding 'machine'. There are 69 moves to KI and 38 from KI, that is, a positive score (surplus). The matrix shows that large universities are generally demanders and to a lower extent suppliers of people to the system. Lund University (LU) and Gothenburg University (GU) both have a positive score (surplus) while Umeå University (UMU), Stockholm University (SU) and KTH (Royal Institute of Technology) have a negative score (deficit). The Institute Sector seems to be a neutral player in this field of research. It receives as many as it supplies, which indicates that it has a stable situation regarding financing of research.

\section{Searching for trends in mobility}

The next step is to utilize the data for a study of traits of mobile persons and the direction of their mobility. We are dealing with the Swedish research landscape and the competition between universities to find competence and excellence. Some are winners and some are losers. Is it possible to put this into figures? Table 8 shows the result of this analysis, which

Table 7. Matrix showing mobility between institutions (all data not shown)

\begin{tabular}{|c|c|c|c|c|c|c|c|c|c|c|c|c|c|c|c|c|c|c|c|c|}
\hline FROM/TO & $\frac{7}{\uparrow}$ & 穴 & 昂 & 资 & 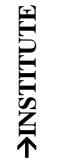 & D્ઞ & $\begin{array}{l}\text { 岕 } \\
\text { 來 }\end{array}$ & 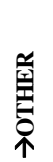 & P & 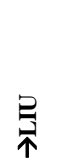 & 光 & 皃 & 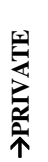 & 里 & 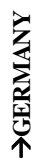 & 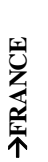 & $\frac{\mathbb{T}}{\mathbb{T}}$ & 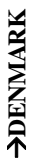 & $\sum_{\infty}$ & 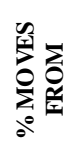 \\
\hline $\begin{array}{l}\text { NO. OF } \\
\text { PEOPLE }\end{array}$ & 140 & 75 & 68 & 45 & 39 & 36 & 28 & 26 & 24 & 15 & 8 & 8 & 7 & 5 & 5 & 3 & 3 & 2 & & \\
\hline $\mathrm{KI} \rightarrow$ & (490) & 6 & 2 & 4 & 7 & 2 & 1 & 6 & 4 & 2 & 0 & 0 & 1 & 0 & 0 & 0 & 1 & 0 & 38 & $36 \%$ \\
\hline $\mathrm{UU} \rightarrow$ & 15 & (224) & 7 & 1 & 9 & 0 & 8 & 3 & 1 & 1 & 0 & 0 & 2 & 2 & 0 & 0 & 0 & 0 & 50 & $62 \%$ \\
\hline $\mathrm{LU} \rightarrow$ & 2 & 1 & (194) & 0 & 1 & 3 & 2 & 2 & 0 & 2 & 2 & 0 & 2 & 0 & 0 & 0 & 0 & 2 & 19 & $36 \%$ \\
\hline $\mathrm{UMU} \rightarrow$ & 5 & 2 & 1 & (120) & 3 & 1 & 3 & 2 & 2 & 0 & 0 & 0 & 1 & 0 & 0 & 1 & 0 & 1 & 25 & $54 \%$ \\
\hline INSTITUTE $\rightarrow$ & 10 & 6 & 5 & 4 & (36) & 2 & 3 & 1 & 1 & 0 & 1 & 0 & 0 & 0 & 0 & 0 & 0 & 0 & 34 & $50 \%$ \\
\hline $\mathrm{GU} \rightarrow$ & 2 & 0 & 1 & 1 & 0 & (124) & 1 & 0 & 0 & 0 & 0 & 0 & 0 & 0 & 0 & 0 & 0 & 0 & 6 & $33 \%$ \\
\hline USA $\rightarrow$ & 4 & 6 & 3 & 1 & 4 & 1 & (8) & 2 & 2 & 0 & 1 & 0 & 0 & 0 & 0 & 0 & 1 & 0 & 26 & $54 \%$ \\
\hline OTHER $\rightarrow$ & 9 & 3 & 4 & 1 & 2 & 1 & 1 & (12) & 0 & 1 & 0 & 0 & 0 & 0 & 0 & 0 & 0 & 0 & 22 & $54 \%$ \\
\hline $\mathrm{SU} \rightarrow$ & 6 & 1 & 1 & 3 & 0 & 0 & 2 & 1 & (50) & 1 & 1 & 0 & 0 & 0 & 0 & 0 & 0 & 0 & 16 & $57 \%$ \\
\hline $\mathrm{LIU} \rightarrow$ & 3 & 0 & 0 & 1 & 0 & 1 & 0 & 1 & 0 & (28) & 0 & 0 & 0 & 0 & 0 & 0 & 0 & 0 & 6 & $46 \%$ \\
\hline $\mathrm{UK} \rightarrow$ & 3 & 0 & 2 & 1 & 1 & 0 & 0 & 0 & 1 & 0 & $(0)$ & 0 & 0 & 0 & 0 & 0 & 0 & 0 & 8 & $62 \%$ \\
\hline FINLAND $\rightarrow$ & 1 & 0 & 1 & 0 & 4 & 0 & 0 & 1 & 0 & 0 & 0 & (20) & 0 & 0 & 1 & 0 & 0 & 0 & 8 & $100 \%$ \\
\hline PRIVATE & 1 & 2 & 2 & 1 & 1 & 0 & 0 & 0 & 0 & 0 & 0 & 0 & (6) & 0 & 0 & 0 & 0 & 0 & 7 & $54 \%$ \\
\hline SLU & 1 & 1 & 0 & 0 & 0 & 0 & 0 & 0 & 0 & 0 & 0 & 0 & 0 & (16) & 0 & 0 & 0 & 0 & 2 & $50 \%$ \\
\hline GERMANY $\rightarrow$ & 1 & 1 & 2 & 1 & 0 & 0 & 0 & 0 & 0 & 0 & 0 & 0 & 0 & 0 & (2) & 0 & 0 & 0 & 5 & $83 \%$ \\
\hline FRANCE $\rightarrow$ & 2 & 0 & 0 & 0 & 0 & 0 & 0 & 0 & 0 & 0 & 0 & 0 & 0 & 0 & 0 & $(0)$ & 0 & 0 & 2 & $67 \%$ \\
\hline $\mathrm{KTH} \rightarrow$ & 0 & 0 & 0 & 1 & 0 & 0 & 1 & 0 & 0 & 0 & 0 & 0 & 0 & 0 & 0 & 0 & (2) & 0 & 3 & $60 \%$ \\
\hline DENMARK $\rightarrow$ & 0 & 1 & 2 & 0 & 0 & 0 & 0 & 0 & 0 & 0 & 0 & 0 & 0 & 0 & 0 & 0 & 0 & $(0)$ & 3 & $50 \%$ \\
\hline SUM & 69 & 31 & 34 & 21 & 34 & 12 & 22 & 19 & 12 & 7 & 5 & $\mathbf{0}$ & 6 & 2 & 1 & 1 & 2 & 3 & & \\
\hline
\end{tabular}

Source: Data from $326 \mathrm{CVs}$

Note: $\quad$ Figures show number of moves between institutions 
Table 8. Mobility and citation rate (CPP/FCS)

\begin{tabular}{|c|c|c|c|c|c|c|c|c|}
\hline & $\rightarrow \mathrm{KI}$ & $\rightarrow \mathrm{UU}$ & $\rightarrow \mathrm{LU}$ & $\rightarrow$ UMU & $\rightarrow \mathrm{GU}$ & $\rightarrow \mathrm{SU}$ & $\rightarrow$ LIU & AVG CPP/FCS $\rightarrow$ \\
\hline $\begin{array}{l}\text { NO. OF } \\
\text { PEOPLE }\end{array}$ & 140 & 75 & 68 & 45 & 36 & 24 & 15 & \\
\hline KI $\rightarrow$ & [1.53 (73)] & $1.31(3)$ & $1.32(2)$ & $1.64(1)$ & $2.08(1)$ & $1.15(2)$ & $0.93(1)$ & $1.41(15)$ \\
\hline UU $\rightarrow$ & $1.59(15)$ & [1.57 (28)] & $1.15(3)$ & $1.08(1)$ & $1.58(2)$ & - & $1.73(1)$ & $1.50(29)$ \\
\hline $\mathbf{L U} \rightarrow$ & - & - & [1.22 (43)] & - & $0.72(2)$ & - & $1.4(2)$ & $1.06(4)$ \\
\hline UMU $\rightarrow$ & - & $1.98(2)$ & $1.02(2)$ & [1.35 (20)] & $1.39(2)$ & $3.55(1)$ & - & $1.77(12)$ \\
\hline GU $\rightarrow$ & $1.98(1)$ & - & - & $0.64(1)$ & [1.16 (22)] & - & - & $1.68(3)$ \\
\hline $\mathrm{SU} \rightarrow$ & $0.90(4)$ & $0.75(1)$ & $1.64(1)$ & $1.66(2)$ & - & [1.03 (5)] & - & $1.23(10)$ \\
\hline LIU $\rightarrow$ & - & - & $0.90(1)$ & - & - & - & [1.29(5)] & $0.90(3)$ \\
\hline $\begin{array}{l}\rightarrow \text { AVG } \\
\text { CPP/FCS }\end{array}$ & $1.68(45)$ & $1.45(10)$ & 1.40 (19) & 1.35 (8) & $1.60(9)$ & 1.48 (6) & $1.36(4)$ & \\
\hline
\end{tabular}

Note: Row summary and column summary report data not shown in the table

indicates the number of persons moving and the performance figure (CPP/FCS) for these researchers.

Table 8 should be read in the following manner. A group of researchers start their career at KI and they are still there or have done a career move to other places. This group of 73 persons have a CPP/FCS of 1.49 , which is $49 \%$ above average. Uppsala (UU) loses a group of 15 researchers that now have appointments at KI. This group is considered as high performers with a CPP/FCS of 1.59. Uppsala has a large group that stays or comes back to UU and these 'local heroes' are also very good performers (1.57).

The bottom-row line gives the average CPP/FCS for all 45 persons who have moved to KI, etc. In the last column we find the other end of the spectrum: all persons moving from KI, etc. Note that these figures report all the mobility to and from these universities. Comparing the means of citation rates makes it possible to draw some preliminary conclusions. Karolinska is the big winner in the competition for human capital in Swedish medical research. Uppsala loses people with 1.50 and receives human capital with 1.30. The same applies to Umeå. Lund University has a positive exchange and this is also the case for Linköping University and Stockholm University. Figures for 'local heroes' are often markedly lower than for the mobile group, except for Uppsala.

\section{Cluster groups and mobility}

Finally, we will try to integrate cluster analysis and mobility analysis. Is there a typical path for the different cluster groups? There are many paths, each one unique for that person, but seen as a group there might be some features of mobility figures that could be of interest. It should be noted that we are investigating mobility in relation to where researchers get their $\mathrm{PhD}$ and where they end up as professors. A first conclusion would be that mobility does not yield any significant differences between male and female investigators.
Group 1 has been characterized as the mobility group. When we dig deeper into the data we find that there is a large group of foreign scientists in this group with a $\mathrm{PhD}$ from abroad. They tend to move around in Europe before finding themselves a permanent position.

Going further to Group 2 we find that there are a few typical paths. This is the immobile group. Their most typical career path starts in Lund or Umeå and ends at the same university. This reflects the low mobility of researchers in Sweden.

Group 3 are the most prestigious researchers, characterized by their excellence, seniority, and 'watchdog' experience. Starting their career at Karolinska or Uppsala, also this group tend to end up at their mother university. A short time period between $\mathrm{PhD}$ and professorship does not seem to be connected to an overall pattern of mobility as this group, after a short career tour, often are recruited back to their home university.

Lastly, we have the mobility of the entrepreneurial group. Here we should notice that postdoc experience differentiates between groups. In general, about half of each group have done a postdoc abroad, mainly in the United States. The entrepreneurial group deviates from this quite strong rule: to a larger extent, about $75 \%$, they have been abroad during their postdoc. They tend to stay longer, but when they come back to Sweden they find themselves a position at the best institutions: Uppsala University and Karolinska Institute.

In conclusion, as soon as we want to use large numbers we always end up with the large group of 'local heroes' - researchers who receive their $\mathrm{PhD}$ at one university and end up as professors at the same university. And this overall pattern does not seem be gender-specific.

\section{Discussion}

The main contribution of this paper is to include citation analysis and test it on a fairly large dataset 


\section{Using field-normalized citations and papers during a specific period of time have given new insights and new perspectives on the problems of gender, mobility, collaboration and productivity}

of CVs. Using field-normalized citations and papers during a specific period of time have given new insights and new perspectives on the problems of gender, mobility, collaboration and productivity. A first result is that gender is related to productivity in a double way: female scientists may be less productive in both normal and fractional paper counts, but their impact in field-normalized citations is better than that of their male colleagues. This is the paradox of gendered productivity patterns that seems to be relevant in many areas of research.

By applying cluster analysis we have been able to pinpoint some of the patterns underlying medical research in Sweden. The four groups of researchers - mobile, immobile, excellence and entrepreneur - reveal important differences when it comes to variables like gender and impact of research. Female scientists are over-represented in the first two groups and under-represented in the latter groups. One implication is that relatively fewer female researchers have the type of resources that are assembled by their male colleagues in the excellence and entrepreneur groups. Well-known Matthew Effects probably lie behind and can explain why this is the case.

The female tendency to lower publication activity but higher citation rates makes it necessary to find ways of translating their revealed excellence into strategies for resource assembling. This they will have to do in relation to research foundations and research councils that, up to now, have been predominantly interested in numbers of publications (Brouns, 2000; Sandström and Hällsten, 2008). In the pessimistic view, female researchers will not be able to assemble resources for their research. Instead, they will have to align with older and maybe not so innovative male colleagues who control the funding resources and still are the main recipients of significant funding resources.

Collaborative research is productive but less collaborative research is cited relatively more frequently. This result goes against many of the propositions of earlier research. Lee and Bozeman (2005) hold the door open for conclusions like these as they do not have a qualitative indicator, and the conclusion here is that these fundamental questions should be further investigated. Mobility between Swedish universities seems to be determined to a large extent by researcher impact (quality) and reputation.

\section{References}

Brouns, M 2000. The gendered nature of assessment procedures on scientific research funding: the Dutch case. Higher Education in Europe, 25, 193-199.

Cañibano, C 2008. Measuring and assessing researcher mobility from CV analysis: the case of the Ramon y Cajal programme in Spain. Research Evaluation, 17(1), March, 17-31.

Cesaroni, F and A Gambardella 2003. Research productivity and the allocation of resources in publicly funded research programmes. In Science and Innovation: Rethinking the Rationales for Funding and Governance, eds. A Geuna et al, pp. 202-232. Cheltenham, UK: Edward Elgar.

Cole, J R and H Zuckerman 1984. The productivity puzzle: persistence and change in patterns of publication of men and women scientists. In Advances in Motivation and Achievement, eds. Maehr and Steinkamp, pp. 217-256. Greenwich: JAl Press.

Dietz, J S and B Bozeman 2005. Academic careers, patents, and productivity: industry experience as scientific and technical human capital. Research Policy, 34, 349-367.

Dietz, J S, I Chompalov, B Bozeman, E O Lane and J W Park 2000. Using the curriculum vita to study the career paths of scientists and engineers: an exploratory assessment. Scientometrics, 49, 419-442.

Gaughan, M and B Bozeman 2002. Using curriculum vitae to compare some impacts of NSF research grants with center funding. Research Evaluation, 11(1), April, 17-26.

Gaughan, M and S Robin 2004. National science training policy and early scientific careers in France and the United States. Research Policy, 33, 569-581.

Glänzel, W 1996. The need for standards in bibliometric research and technology. Scientometrics, 35,167-176.

Lee, S and B Bozeman 2005. The impact of research collaboration on scientific productivity. Social Studies of Science, 35(5), 673-702.

Long, J S 1992. Measures of sex differences in scientific productivity. Social Forces, 71(1), 159-178.

Mangematin, V 2000. PhD job market: professional trajectories and incentives during the PhD. Research Policy, 29(6), 741-756.

Mangematin, V 2001. Individual careers and collective research: is there a paradox? International Journal of Technology Management, 22(7-8), 670-675.

Merton, R K 1973. The Sociology of Science: Theoretical and Empirical Investigations. Chicago: Chicago University Press.

Narin, F and K S Hamilton 1996. Bibliometric performance measures. Scientometrics, 36(3), 293-310.

Nederhof, A J and M S Visser 2004. Quantitative deconstruction of citation impact indicators: waxing field impact but waning journal impact. Journal of Documentation, 60(6), 658-672.

Prpic, K 2002. Gender and productivity differentials in science. Scientometrics, 55(1), 27-58.

Sandström, U and M Hällsten 2008. Persistent nepotism in peer review. Scientometrics, 74(2), 175-189.

Sandström, U and M Hällsten 2009. Research quality and diversity of funding: a model for relating research money to output of research. Scientometrics. DOI 10.1007/s11192-009-0422-2.

Tijssen, R J W and J de Leeuw 1988. Multivariate data-analysis methods in bibliometric studies of science and technology. In Handbook of Quantitative Studies of Science and Technology, ed. A F J van Raan, pp. 705-740. Amsterdam: Elsevier Science Publishers.

Van Raan, A F J 2004. Measuring science. In Handbook of Quantitative Science and Technology Research, eds. H F Moed, W Glänzel and U Schmoch, pp. 19-50. Dordrecht: Kluwer Academic Publishers.

Van Raan, A F J 2006. Statistical properties of bibliometric indicators: research group indicator distributions and correlations. Journal of the American Society for Information Science and Technology, 57(3), 408-430.

Xie, $Y$ and $K$ A Shauman 1998. Sex differences in research productivity: new evidence about an old puzzle. American Sociological Review, 63(6), 847-870. 
Copyright of Research Evaluation is the property of Beech Tree Publishing and its content may not be copied or emailed to multiple sites or posted to a listserv without the copyright holder's express written permission. However, users may print, download, or email articles for individual use. 